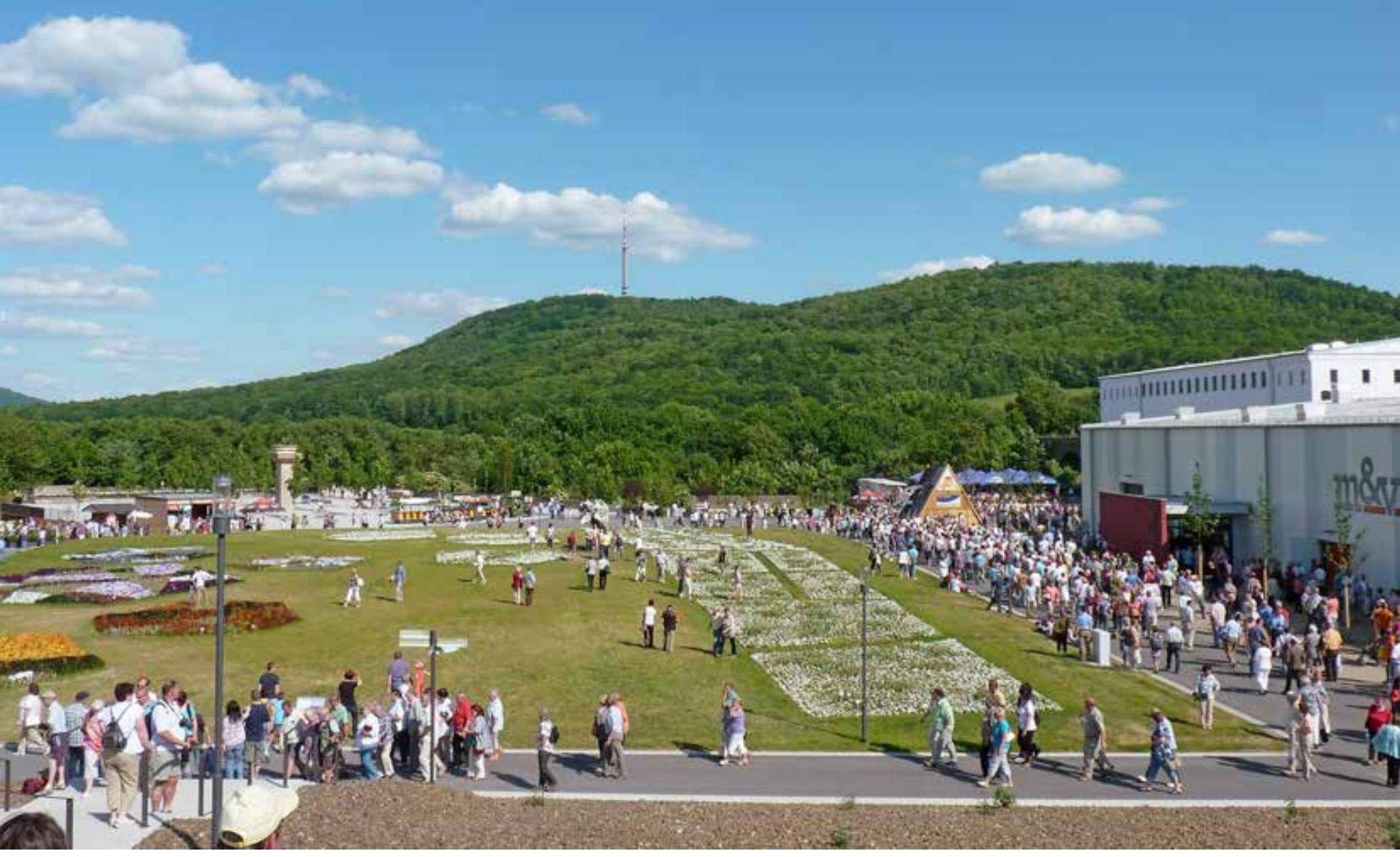

\title{
Vom Industriestandort zum modernen Veranstaltungszentrum und Naherholungsgebiet
}

\author{
Philipp Zirps
}

Nach der Schließung der ehemaligen Zuckerfabrik am 31. Dezember 2002 entwickelte sich in der Großen Kreisstadt Löbau die Idee, die Brachflächen des ehemaligen Standortes sowie des ehemals industriell genutzten Talraumes des Löbauer Wassers ökologisch aufzuwerten. Auf einer Gesamtfläche von ca. 20 Hektar sollte ein dauerhaft grüner Erholungs- und Erlebnisbereich geschaffen werden. Mit dem Stadtratsbeschluss vom 5. Juli 2007 reichte die Große Kreisstadt Löbau ihre Bewerbung für die Ausrichtung der 6. Sächsischen Landesgartenschau 2012 beim Sächsischen Staatsministerium für Umwelt und Landwirtschaft ein. Bereits fünf
Monate später beschloss das sächsische Kabinett am 11. Dezember 2007, die 6. Sächsische Landesgartenschau nach Löbau zu vergeben.

Die Vision der Stadt Löbau war es, mit vielen Partnern und den Löbauer Bürgern im Rahmen der Stadtentwicklung, auf einem ehemaligen Industriebrachengelände eine grüne Oase und einen Veranstaltungspark am Fuße des Löbauer Berges zu gestalten. Was dann im Zuge der Vorbereitungen und der Durchführung der Landesgartenschau geschaffen wurde, hat teilweise die Erwartungen und Phantasien übertroffen. Doch vor der Umgestaltung musste ein Konzept erarbeitet werden, wofür ein Wettbewerb ausge-
Blick vom Haupteingang über das Zuckerplateau auf den Löbauer Berg

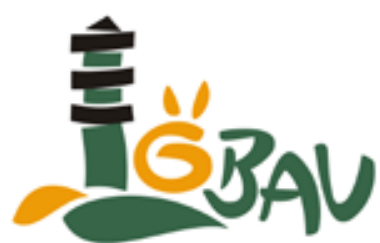

LandesGartenSchau LÖBAU 2012 28. April14.0ktober 


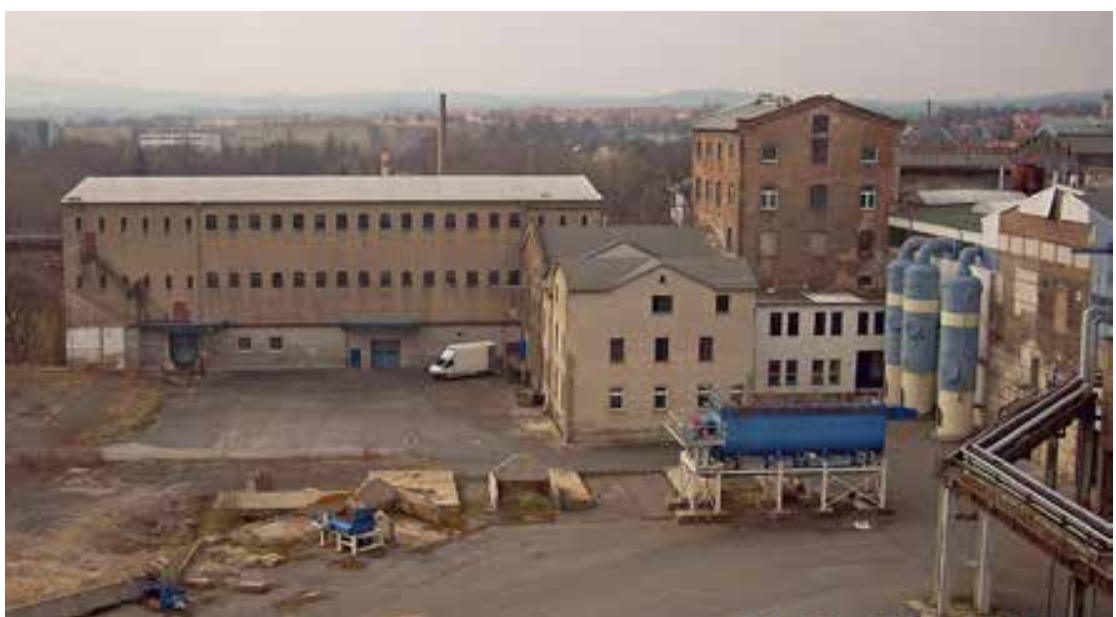

Blick auf das Gelände der ehemaligen Zuckerfabrik schrieben wurde. Unter allen Teilnehmern hatte das Landschaftsarchitekturbüro hutterreimann+ cejka die Vision der Stadt am besten dargestellt und umgesetzt. Deshalb war das Büro im Landschaftsarchitektonischen Ideen- und Realisierungswettbewerb zur 6. Sächsischen Landesgartenschau Löbau 2012 verdienter Preisträger des 1. Preises. Zur Preisverleihung im Jahr 2008 sagte Oberbürgermeister Dietmar Buchholz, dass die Stadt Löbau sich sicher sei, dass für Löbau die bestmögliche Variante mit dem Konzept der Landschaftsarchitekten hutterreimann+cejka „gekürt“ wurde. Diese Worte sollten noch einmal von großer Bedeutung sein. Denn das Architekturbüro wurde für das Projekt „Park am Löbauer Wasser - Landesgartenschau Löbau 2012“ mit dem Deutschen LandschaftsarchitekturPreis 2013 ausgezeichnet.

\section{Der Beginn eines Rekultivierungsprozesses}

Nachdem bereits im Sommer 2009 mit den ersten Abbruch - und Tiefenenttrümmerungsmaßnah- men Altbestände der Industriezweige entfernt wurden, begann mit einem offiziellen Spatenstich am 23. April 2010 die Bauausführung der Neubaumaßnahmen zur Landesgartenschau und dem damit verbundenen Rekultivierungsprozess des Geländes. Die Maßnahme der „Revitalisierung des Geländes der ehemaligen Zuckerfabrik - Tiefenenttrümmerung“ wurde im Rahmen eines Programms des Freistaates Sachsen (EFRE-Programm) gefördert. Mit einem Investitionsvolumen von ca. zwölf Millionen Euro entstand in den folgenden beiden Jahren bis zur Eröffnung der 6 . Sächsischen Landesgartenschau am 28. April 2012 ein nachhaltiges, nutzbares Naherholungsgebiet. Mit dem Bau einer 4.000 Quadratmeter großen Messe - und Veranstaltungshalle im Jahr 2011 wurde zusätzlich ein infrastrukturell starkes und leistungsfähiges Veranstaltungszentrum geschaffen, welches im Nachnutzungskonzept einen wichtigen Bestandteil darstellen sollte.

Im ursprünglichen Entwurf der hutterreimann Landschaftsarchitektur GmbH war der Bau einer solchen Eventhalle nicht vorgesehen. Erstmalig in der Geschichte der sächsischen Landesgartenschauen konnten nun Konzerte, Shows und Ausstellungen in einem festen Veranstaltungskomplex durchgeführt werden.

\section{„Auf kurzem Weg ins Grüne“}

Unter diesem Motto vermarktete die Landesgartenschau Löbau gGmbH sowie die Stadt Löbau das entstandene Gartenschaugelände. Vom 28. April 2012 bis zum 14. Oktober 2012 wurden über 480.000 Besucher bei der 6. Sächsischen Landesgartenschau in der Stadt am Berge begrüßt.

Mit dem Blick auf die einmalige Landschaft des Löbauer Berges und seinem Gusseisernen Aus-

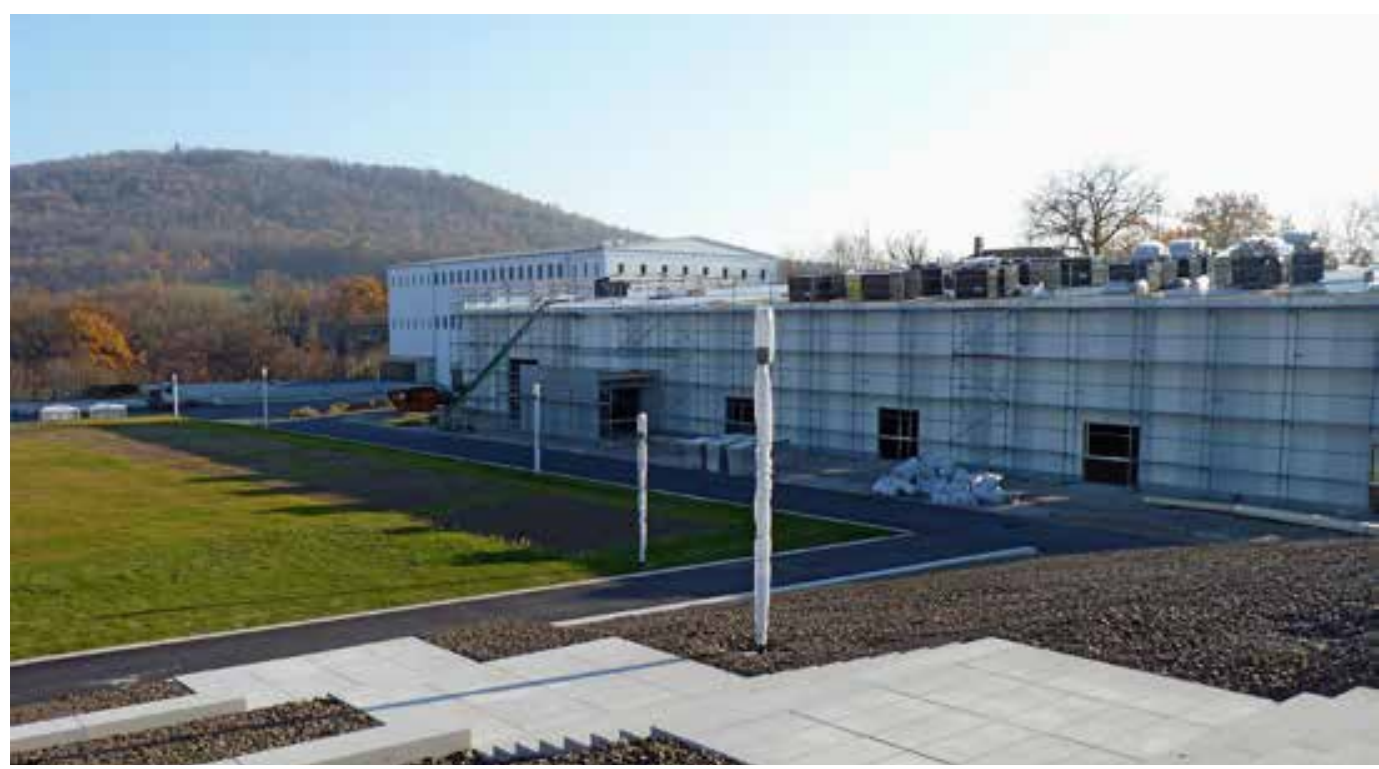


sichtturm sowie dem Blütenmeer der bepflanzten Wechselfloorflächen im Eingangsbereich des Gartenschaugeländes, erlebten die Besucher im ersten Augenblick den besonderen Charme des Standortes. Allein für die Frühjahrsbepflanzung wurden auf dem gesamten Gelände insgesamt über 105.000 Frühjahrsblüher wie Hornveilchen, Stiefmütterchen, weißer Mohn oder auch Tulpen angelegt.

Wechselnde Bepflanzungen fanden die Besucher auch bei den insgesamt vierzehn stattfindenden Blumenschauen in dem ehemaligen Zuckerlager, der sogenannten Blumenhalle, wieder. Im gesamten Veranstaltungszeitraum wurden über 500 Unterhaltungsprogramme verschiedenster Art mit ca. 4.000 Mitwirkenden in der Messe und Veranstaltungshalle sowie auf dem Freigelände aufgeführt. Höhepunkte waren unter anderem die Konzerte von Andreas Martin und Frank Zander, 30 Jahre „Traumzauberbaum“ mit dem Reinhard Lakomy Ensemble sowie die Eröffnungs- und Abschlussveranstaltung.

Mit der Abschlussveranstaltung am 14. Oktober 2012 schlossen sich vorerst wieder die Tore des Geländes. Das dargebotene Potential und der Zuspruch der Bevölkerung unserer Region waren Anlass und zugleich ein Antrieb um ein erfolgreiches und nachhaltiges Nachnutzungskonzept zu entwickeln.

\section{Messe- und Veranstaltungspark Löbau}

Das Nachnutzungskonzept sah vor, ein modernes Veranstaltungszentrum mit der größten Ver-

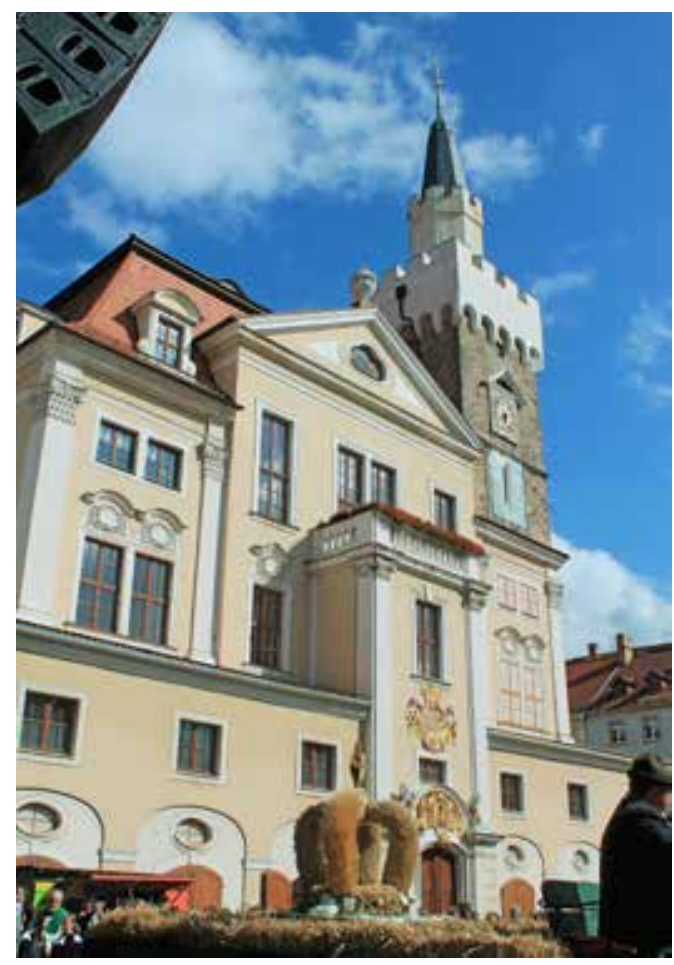

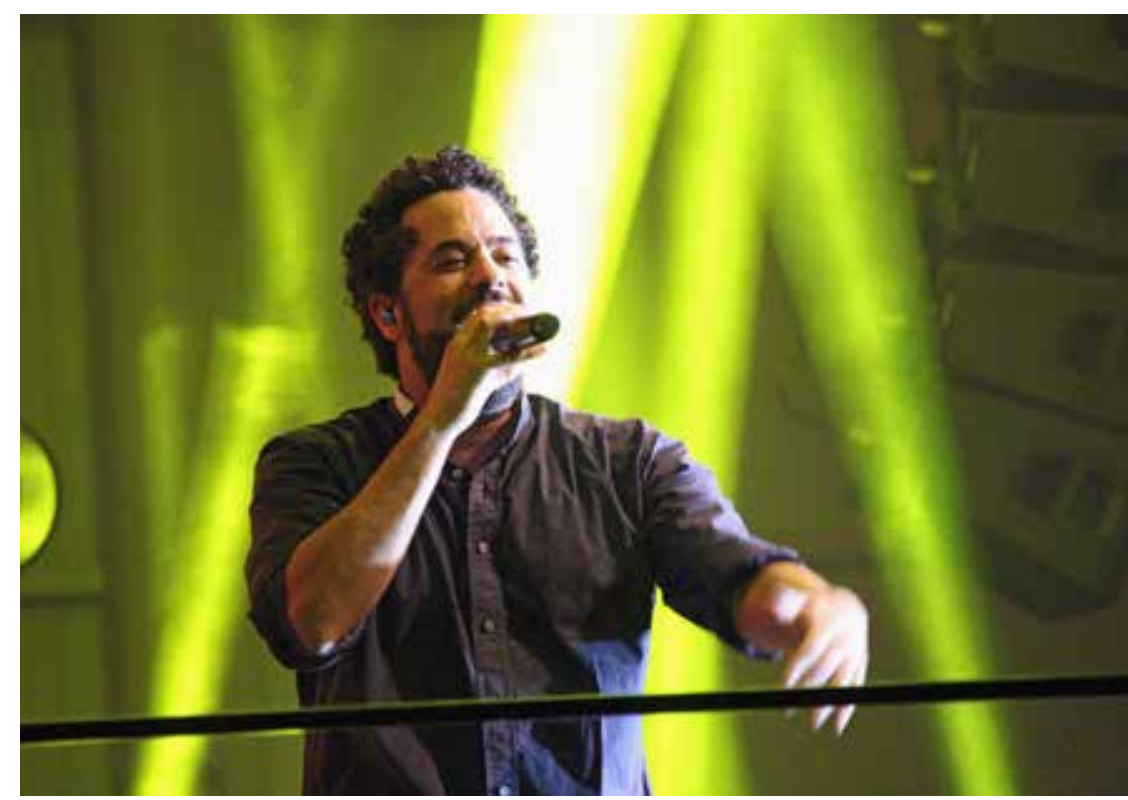

anstaltungshalle zwischen Dresden und Breslau, unter der Geschäftsführung von Joachim Birnbaum im Landkreis Görlitz und darüber hinaus zu etablieren. Bis heute gab es keine Umfirmierung, so dass die Geschäftstätigkeit des Messeund Veranstaltungsparks als Landesgartenschau Löbau gGmbH ausgeübt wird.

Bereits Anfang März 2013 wurden die ersten Veranstaltungen von Ausstellungen über Konzerte bis hin zu Messen in der Messe - und Veranstaltungshalle sowie der Blumenhalle organisiert. Nachdem die Resonanz sehr positiv ausfiel und die Nachfrage kontinuierlich stieg, konnte an der Fortführung des entwickelten Konzeptes festgehalten und gearbeitet werden.

\section{„Das Wunder im Pollunder“}

Damit den Besuchern eine Vielfalt geboten wird und die entstandene Nachfrage eine Bedürfnisbefriedigung erfahren konnte, wurden neue Genres im Messe- und Veranstaltungspark angeboten. Mit dem „Wunder im Pollunder“ wurde durch Olaf Schubert im März 2014 das Genre „Comedy“ in Löbau wiederbelebt. Die Nachfrage war so groß, dass mit 3.100 Sitzplätzen die Kapazitätsgrenze der Halle erreicht und eine zusätzliche Show organisiert wurde. Als Kennzahlen für das Wachstum kann die Veranstaltungsdichte sowie die Besucherzahl betrachtet werden. Im Vergleich zum Vorjahr lag das Wachstum der Veranstaltungsdichte mit über 100 Veranstaltungen in 2014 bei $200 \%$. Auch die Besucherzahlen verdeutlichen ein starkes Wachstum von 60.000 in 2013 auf über 100.000 in 2014.

Zudem wurde durch die Einstellung von Frau Weiß als Veranstaltungsmanagerin die perso-
Konzert von Adel Tawil zum Landeserntedankfest 2015
Erntekrone vor dem Löbauer Rathaus zum Landeserntedankfest 2015 
Panorama der Gewerbemesse Konvent'a mit Blick auf den Löbauer Berg

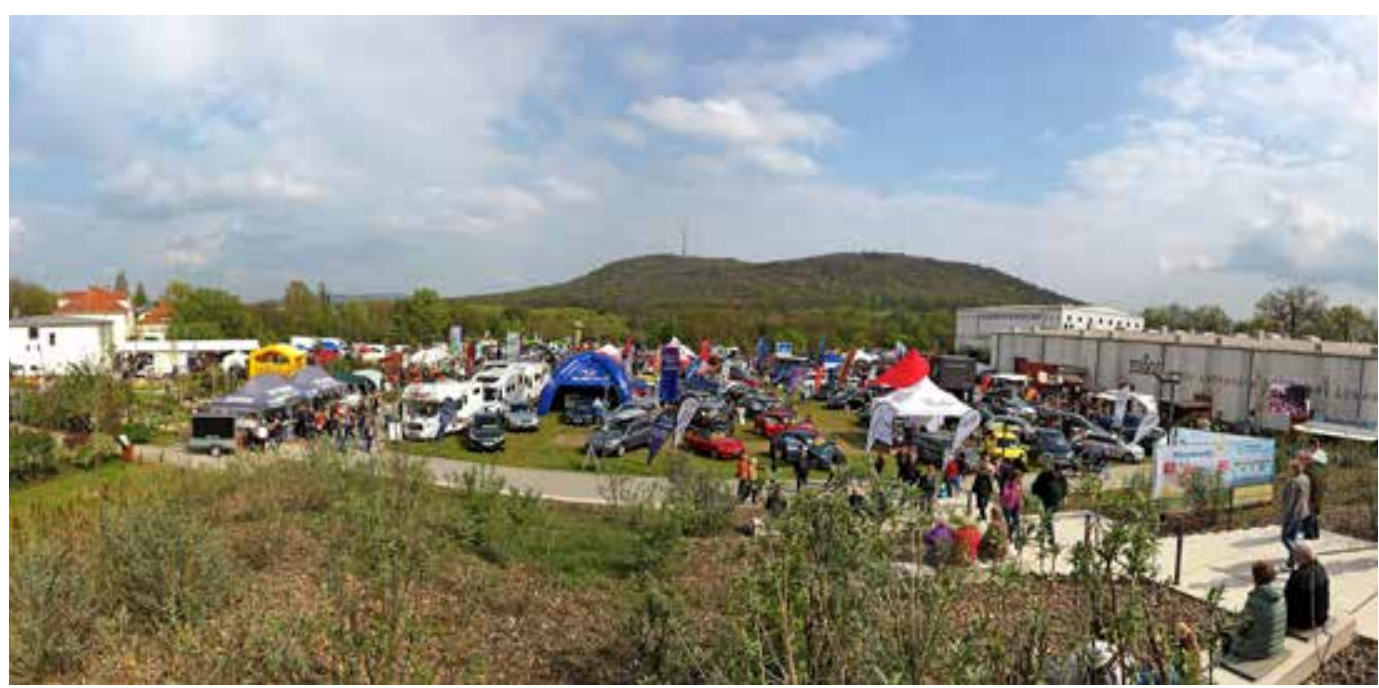

nelle Struktur des Unternehmens gesichert und ausgebaut.

\section{Erntedank in Löbau}

Im Jahr 2015 wurde die Geschichte des erfolgreichsten Nachnutzungskonzeptes aller Sächsischen Landesgartenschauen fortgesetzt. Höhepunkt in diesem Jahr war das 18. Sächsische Landeserntedankfest vom 18. bis 20. September 2015, welches gleichzeitig als Generalprobe für den Tag der Sachsen 2017 in Löbau angesehen wurde. Aufgrund idealer Standortfaktoren, Erfahrungswerten der Verantwortlichen vor Ort sowie einem kulturellen Rahmenprogramm der Extraklasse, gespickt mit Stars wie Adel Tawil oder Electra, wurden an dem Festwochenende über 80.000 Besucher auf dem Gelände sowie der Innenstadt Löbaus begrüßt.

\section{Jahre Gewerbemesse Konvent'a}

Als Austragungsort und Veranstalter der größten Leistungs- und Gewerbeschau in der Oberlausitz wurde im April 2016 auf dem Messeparkgelände das 15-jährige Jubiläum der Gewerbemesse Konvent'a gefeiert. Seit 2014 wirken die Landesgartenschau Löbau gGmbH und der Konvent'a Verein gemeinsam an der Durchführung der größten Messe in der Region. Durch die Erfolgsstrategie der Leitmesse des Messeparks konnten in den Folgejahren weitere Messen wie unter anderem die Doppelmesse „Feiern \& Genießen“ und „Reisen \& Vital“, die Baumesse Löbau, die Modellbahnausstellung Görlitz + Löbau oder auch die Ausbildungsmesse INSIDERTREFF für den Standort gewonnen werden. Die Gewerbemesse Konvent'a verdeutlicht Jahr für Jahr die Wirtschaftskraft und das Potential der Unternehmen in der Region. Die Besucher können sich im direkten Kontakt über Produkte oder auch Stellenangebote informieren. Generell hat sich der Bereich „Messe“ seit 2013 zu einem wichtigen Standbein etabliert. 2016 fanden 14 verschiedene Messen/Ausstellungen mit ca. 90.000 Besuchern statt. Im Vergleich waren 2013 ca. 40.000 Besucher bei sechs Messen/ Ausstellungen

\section{Jahre Messe- und Veranstaltungshalle Löbau}

Am 28. April 2017 wurde mit fünf Jahren Messeund Veranstaltungshalle ein „kleiner“ Meilenstein erreicht. Die stetig wachsende Veranstaltungsdichte und Besucherzahl in den vergangenen Jahren unterstreicht die erfolgreiche Arbeit der Mitarbeiter des Messeparks und verdeutlicht die Unterstützung der ansässigen Einwohner, Unternehmen und Partner der Region. Ohne die tatkräftige Unterstützung von Veranstaltungsagenturen und externen Partnern könnte ein solches Nachnutzungskonzept nicht erhalten werden.

\section{Tag der Sachsen 2017}

Mit der Ausrichtung des 26. Tages der Sachsen 2017 in Löbau wird auch der Messe- und Veranstaltungspark als ein Teil des Festgebietes wieder eine entscheidende Rolle für die Durchführbarkeit des größten sächsischen Vereins- und Heimatfestes einnehmen.

Neben der Einbindung von zwei Medienpartnern im Bereich der Messe- und Veranstaltungshalle werden im Parkgelände mit der Handwerkermeile, der Sächsischen Tourismusmeile sowie dem Sächsischen Land- und Genussmarkt regionale Themenschwerpunkte integriert.

Falls Sie fragen haben, können Sie uns jederzeit kontaktieren. Wir stehen Ihnen gern mit Rat und Tat zur Seite. 